\title{
SAND REPORT
}

SAND2002-3694

Unlimited Release

Printed November 2002

High Efficiency Optical MEMS by the Integration of Photonic Lattices With Surface MEMS

Authors: James G.Fleming, Shawn-Yu Lin, Seethambal S. Mani, Jeffry Sniegowski, Steven Rodgers and Daryl Dagel

\section{Prepared by}

Sandia National Laboratories

Albuquerque, New Mexico 87185 and Livermore, California 94550

Sandia is a multiprogram laboratory operated by Sandia Corporation,

a Lockheed Martin Company, for the United States Department of Energy's

National Nuclear Security Administration under Contract DE-AC04-94-AL85000.

Approved for public release; further dissemination unlimited.

\section{Sandia National Laboratories}


Issued by Sandia National Laboratories, operated for the United States Department of Energy by Sandia Corporation.

NOTICE: This report was prepared as an account of work sponsored by an agency of the United States Government. Neither the United States Government, nor any agency thereof, nor any of their employees, nor any of their contractors, subcontractors, or their employees, make any warranty, express or implied, or assume any legal liability or responsibility for the accuracy, completeness, or usefulness of any information, apparatus, product, or process disclosed, or represent that its use would not infringe privately owned rights. Reference herein to any specific commercial product, process, or service by trade name, trademark, manufacturer, or otherwise, does not necessarily constitute or imply its endorsement, recommendation, or favoring by the United States Government, any agency thereof, or any of their contractors or subcontractors. The views and opinions expressed herein do not necessarily state or reflect those of the United States Government, any agency thereof, or any of their contractors.

Printed in the United States of America. This report has been reproduced directly from the best available copy.

Available to DOE and DOE contractors from

U.S. Department of Energy

Office of Scientific and Technical Information

P.O. Box 62

Oak Ridge, TN 37831

Telephone: (865)576-8401

Facsimile: (865)576-5728

E-Mail: reports@adonis.osti.gov

Online ordering: http://www.doe.gov/bridge

Available to the public from

U.S. Department of Commerce

National Technical Information Service

5285 Port Royal Rd

Springfield, VA 22161

Telephone: (800)553-6847

Facsimile: (703)605-6900

E-Mail: orders@ntis.fedworld.gov

Online order: http://www.ntis.gov/help/ordermethods.asp?loc=7-4-0\#online

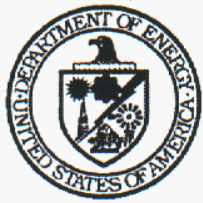




\title{
SAND2002-3694 \\ Unlimited Release \\ Printed November 2002
}

\section{High Efficiency Optical MEMS by the Integration of Photonic Lattices With Surface MEMS}

\author{
James G.Fleming, Shawn-Yu Lin, Seethambal S. Mani, Jeffry Sniegowski, \\ Steven Rodgers and Daryl Dagel \\ MEMS and Novel Silicon Technologies, Sandia National Laboratories \\ P.O. Box 5800, \\ Albuquerque, NM, 87185-1080
}

\begin{abstract}
This report outlines our work on the integration of high efficiency photonic lattice structures with MEMS (MicroElectroMechanical Systems). The simplest of these structures were based on 1-D mirror structures. These were integrated into a variety of devices, movable mirrors, switchable cavities and finally into Bragg fiber structures which enable the control of light in at least 2 dimensions. Of these devices, the most complex were the Bragg fibers. Bragg fibers consist of hollow tubes in which light is guided in a low index media (air) and confined by surrounding Bragg mirror stacks. In this work, structures with internal diameters from 5 to 30 microns have been fabricated and much larger structures should also be possible. We have demonstrated the fabrication of these structures with short wavelength band edges ranging from 400 to $1600 \mathrm{~nm}$. There may be potential applications for such structures in the fields of integrated optics and BioMEMS. We have also looked at the possibility of waveguiding in 3 dimensions by integrating defects into 3dimensional photonic lattice structures. Eventually it may be possible to tune such structures by mechanically modulating the defects.
\end{abstract}




\section{ACKNOWLEDGEMENTS}

The authors gratefully acknowledge the efforts of their coworkers at Sandia National Laboratories Microelectronics Development Laboratory and the Compound Semiconductor Research Laboratory, especially Melanie Tuck and James Bur. 


\section{INTRODUCTION}

There is considerable interest in optical MEMS. However, such devices typically do not take advantage of the full range of possibilities that arise from the wave nature of light. They typically consist of conceptually simple, but structurally complex, mechanical systems, which are really just movable mirrors. On the other hand there has also been considerable progress in the area of Photonic lattice structures. Photonic lattices can be thought of as the Photonic analogue of semiconductors. The photonic band structure results when light encounters a well-defined repeating arrangement of materials with differing refractive index's. This behavior is entirely independent of the electronic properties of the material making up the lattice, as long as it does not strongly adsorb. When correctly designed and fabricated, the most complex of these 3-D structures can exhibit the property that photons with the bandgap energy cannot penetrate the lattice, regardless of their angle of incidence. However, such full three dimensional structures are relatively complex conceptually and structurally and are very difficult to fabricate. There are 1-D and 2-D structures, which are far easier to fabricate and which in some cases can be made to recover many of the properties of the much more complex 3-D structures. This is particularly true of Bragg fiber structures, which form the bulk of this work. The application of Photonic lattice structures to MEMS allows us to enhance the capabilities of MEMS devices and adds functionality to photonic lattices. The size scale of such devices is almost ideal for MEMS; small displacements give rise to large changes in optical properties. MEMS can offer to advanced photonics a simple, manufacturable "switch". On the other hand, advanced photonics offers the ability to not just reflect light in free space, but to confine light within waveguides where it can be efficiently manipulated, switched, and filtered. This added functionality can not be realized by current MEMS technology.

\section{I-D MIRROR}

One dimensional mirror structures were initially fabricated in order to demonstrate proof of concept and compatibility with our current fabrication processes. The initial stacks were fabricated in silicon dioxide and polysilicon. The thickness of each layer corresponded to that of the $1 / 4$ wavelength of light with a wavelength of $\sim 1.5$ microns. The end result of one such run is given in Fig. 1.

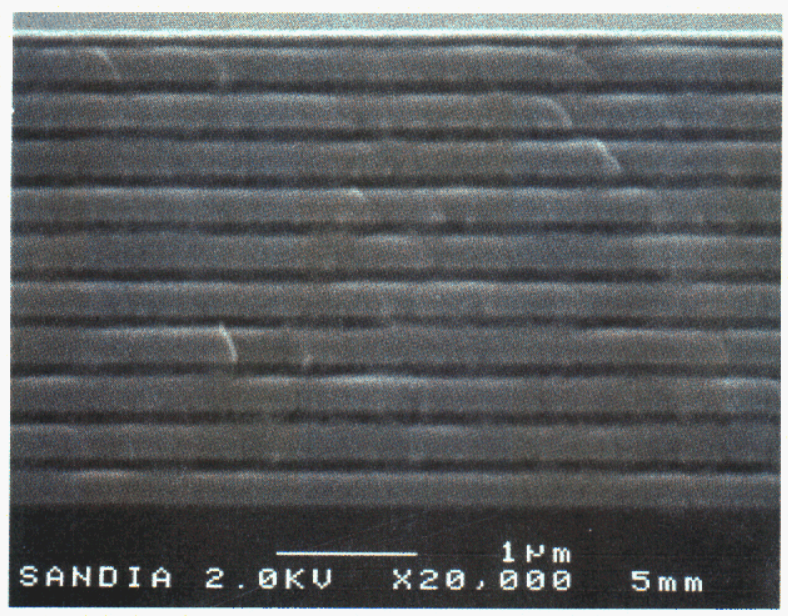

Figure 1 showing an SEM cross section through a mirror stack of polysilicon 1000 angstroms in thickness and silicon dioxide, 2400 angstroms in thickness. As can be seen the layers deposited are smooth and very uniform in thickness. This clearly indicates that silicon processing tools can be used to fabricate such 1-D mirror stacks. 
The properties of these mirror stacks were determined by measuring the reflection spectrum as a function of wavelength. In regions of the band gap there is high reflectivity. As a baseline these mirrors were compared with a more traditional mirror stack grown in III-V material systems. The III-V mirror is sufficient in quality for the creation of a vertical cavity surface emitting laser. As can be seen from Figure 2, the performance of the silicon dioxide/polysilicon mirror stack is superior in performance in terms of bandwidth and reflectivity, even though the silicon based mirror stack consisted of only 5 pairs versus 27 pairs in the case of the III-V stack. This is a result of the higher index of refraction contrast achievable in the silicon-based system. Of course it must also be kept in mind that the silicon-based system is not optically active.

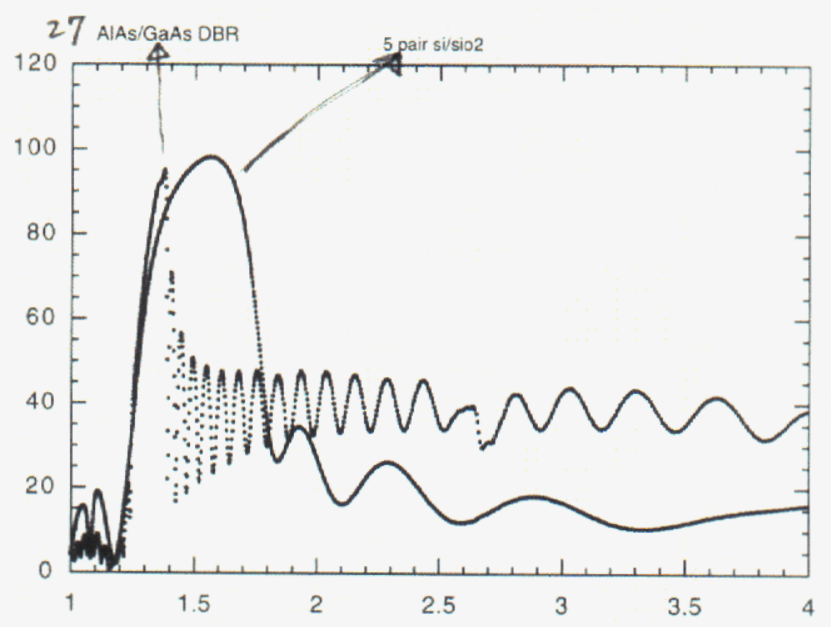

Figure 2. Reflection spectra as a function of wavelength for a silicon/silicon dioxide stack with 5 pairs, versus a 27 pair AlAs/GaAs mirror stack. The reflectivity and band width of the silicon based mirror is greater due to the larger refractive index contrast possible in this system.

\section{1-D CAVITY SWITCH}

Initial work was done on modeling a 1-D cavity switch, in such a structure a resonant cavity is created between a fixed and movable mirror. By changing the cavity dimension it is possible to change the resonant wavelength. Wavelengths, which can resonant in the cavity, will be transmitted while other wavelengths will be reflected. An example of the results obtained is given in Fig. 3. As can be seen from the figure, the width of the resonant peak is sharp, $\sim 1 \mathrm{~nm}$ and there is good contrast between the resonant wavelength and the background. This modeling demonstrated the viability of the pursuit of such a structure.

A diagnostic-based mask layout was designed to develop both a MEMS compatible process flow and to evaluate several variations of the mechanical design of the 1-D photonic switch. The basic design premise for the 1-D switch was to split the 1-D lattice stack allowing the periodicity of the stack to be mechanically modulated between an ON and an OFF transmissive state. The basic concept involved lower fixed layers of the stack comprised of polysilicon and oxide of $1000 \mathrm{~A}$ and $2400 \mathrm{~A}$ thicknesses respectively. This was separated by an air gap from a movable upper stack comprised of polysilicon and low-stress silicon nitride, of 1000A and 1850A respectively. The fixed stack is comprised of 3 polysilicon/oxide pairs, while the movable stack is comprised of 4.5 pairs of 
polysilicon/silicon nitride pairs. The air gap forms when a sacrificial layer of silicon dioxide (4000A thickness) located between the movable and fixed layers is exposed to hydrofluoric acid and is removed. The switching function occurs when the upper stack is moved either closer to or further away from the lower fixed stack. This is accomplished using vertical electrostatic forces applied to the upper stack. Figure 4 illustrates a typical configuration of the switch. The center circular area is the active switch area. This circle, comprised of the upper lattice stack, is suspended from the substrate at a nominal air gap of $4000 \mathrm{~A}$ by the triad of simple spring elements attached at their ends to the supporting substrate. The center circle can either be pulled closer to the lower stack by applying a bias voltage between the movable stack and the substrate layers or levitated by applying bias between one of the lower layers in the movable stack and upper layers in the surrounding perimeter area.

The process flow for the 2-D photonic switch required 2 photomask levels. The first mask is used to define the circular mirror area with its supporting springs by etching through the movable top half-stack of poly-SiN. This exposes the intervening 4000A oxide film allowing the release etch in $\mathrm{HF}$ acid as the final step in the process. A second photomask is used to reduce the vertical thickness of the supporting springs and also allow a better configuration for the levitation.

The switch devices have completed fabrication. Initial results obtained prior to release of the parts indicates a performance very similar to that predicted by theory. However, the spring suspension was found to be too flimsy to be functional. The next configuration consisted of essentially of two $\mathrm{Si} / \mathrm{SiN}$ mirrors stacks separated by a thin oxide with a series of holes etched through the top film in a range of different hole densities. In this configuration there is no spring, the entire suspended membrane flexes. These parts were sufficiently rigid and could be readily actuated below $20 \mathrm{~V}$.

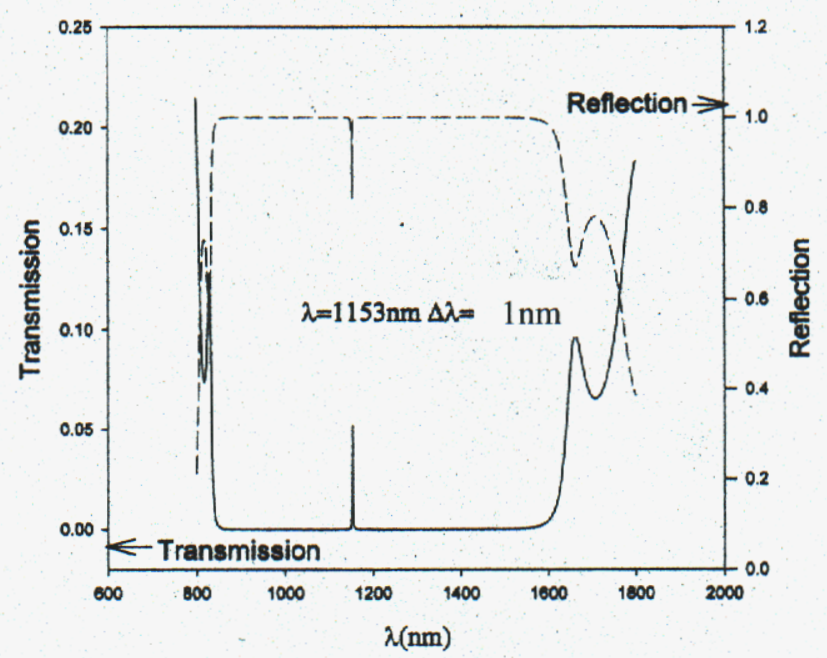

Figure 3. Modeling of movable cavity structure. The width of the resonant peak is on the order of $1 \mathrm{~nm}$. There were 4 mirror pairs, in each mirror segment. 


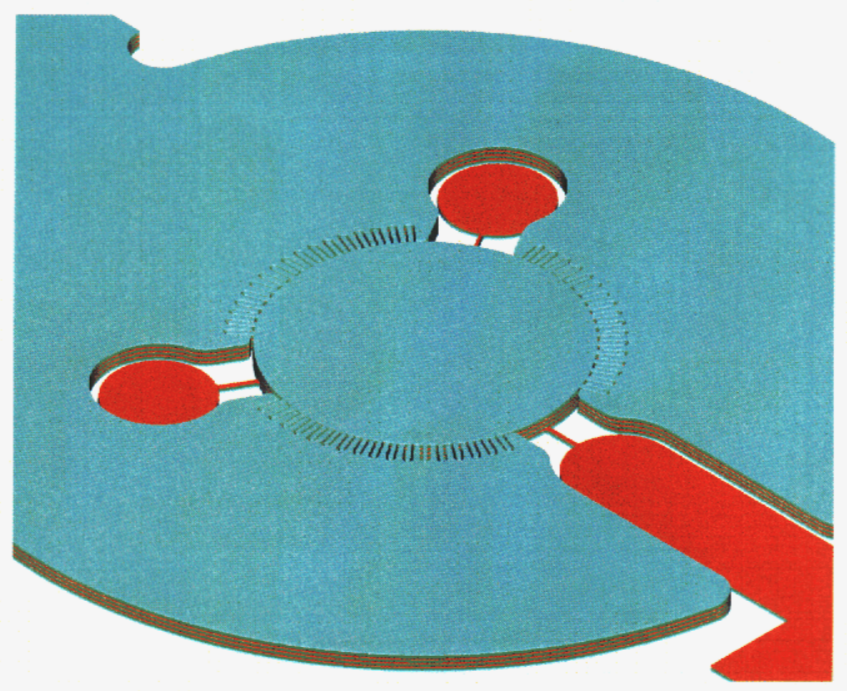

Figure 4. Schematic of one of the initial designs laid out. The movable mirror stack is the large central disc. Electrical contact to the upper electrical contact is achieved by the etched out, red regions. The comb fingers were designed to generate a vertical levitation force when a potential was applied between the outer and inner blue regions.

\section{1-D MIRROR INTEGRATED WITH SUMMIT TM}

The baseline SUMMiT ${ }^{\mathrm{TM}}-\mathrm{V}$ process requires two additional masks to include the 1-D mirror into the various mirror designs being explored. Essentially, the current mirror area comprised of polysilicon surfaces is replaced with the 1-D lattice stack. In order to facilitate the normal "release" process to form the mechanical devices in the SUMMiT ${ }^{\mathrm{TM}}$ MEMS technology, the 1-D micromirror surface needed to be designed within a supporting frame of polysilicon. This invoked the usage of an additional photomask to define the supporting mirror frame structure in the polysilicon layer. The next step requires the definition of the 1-D mirror, requiring the second additional mask. The final definition is for the formation of the final mirror structure in the supporting polysilicon layer, an already existing mask level. A typical mirror layout is shown in figure 4, including the added 1-D lattice mirror area. These mirror devices have full 2-D scanning capability and will benefit greatly from the addition of an integrated high-efficiency mirror targeting the desired wavelengths such as $1.55 \mathrm{um}$.

In order to expedite the attempt to include the 1-D lattice in the standard SUMMiT ${ }^{\mathrm{TM}}-\mathrm{V}$, we utilized an existing photomask set that was used to prove out a sophisticated, 2-D scanning mirror design that used the uppermost polysilicon layer, $\mathrm{P} 4$, as the reflective mirror surface. Normally, this polysilicon mirror surface is coated with a reflective gold layer to enhance reflectivity. However, for several applications, the level of reflectivity is too low and the high-efficiency of the 1-D lattice is desirable. In order to include the 1-D mirror, several issues needed to be addressed. Suitable film choice with high index contrast is necessary, but control of the film mechanical stresses must also be controllable so that fabrication and final mirror configuration is possible. The choice of low-stress silicon nitride and undoped polysilicon fulfilled these requirements. Films stresses were suitably low, therefore, this does not interfere with the operation of the toolset and photodefinition, and the film stack survives the final $\mathrm{HF}$ release etch to produce the movable mirror platforms. Design and addition of the additional two photomasks and modification of the existing P4 mask was completed and a fabrication of this reticle set designated as RS224 is nearly completed. Results to date indicate that the process will now conclude shortly and successfully. These results were in part expediciously executed because of the earlier successes with the fabrication of the 1-D photonics switch. This accomplishment has added significant value of this technology to future application to DP and SBU needs. 
The major problem encountered was mirror stress, this was somewhat surprising since the silicon nitride used was nominally low stress and the silicon was thin and annealed in the normal manner. In the short term this could be addressed by either reducing the mirror diameter or increasing the stiffness of the rim of the mirror to create a "drum" configuration.

\section{DEMONSTRATION OF WAVEGUIDING IN 3-D STRUCTURES}

One of the objectives of this project was to demonstrate the feasibility of waveguiding in 3-D photonic lattice structures. If this is possible, it may feasible to tune and switch such waveguides by mechanically moving certain of the rods making up the lattice. Such a 3-D waveguiding structure was fabricated with a bandgap at $\sim 7$ microns, which was the shortest wavelength achievable at the time. The guide was formed by removing a single rod in the structure; bends were created by intersecting removed rod segments on neighboring levels. The degree of overlap of the removed segments was set at 1/4 of the wavelength, based on modeling done by Iowa State University [Private communication]. An SEM image of the resulting structure is given in Fig. 5. The resulting reflection spectrum is given in Fig. 6. It was necessary to use a reflection technique to analyze the properties of the guide due to the relatively long wavelength of the band gap. In this approach the reflection spectra of a region without the guide is compared with the reflection spectra of a region having a guide. If light is propagating through the waveguide it will be absent in the reflection spectra associated with the waveguide. This is found to be the case at $\sim 7$ microns which is consistent with the bandgap of the structure.

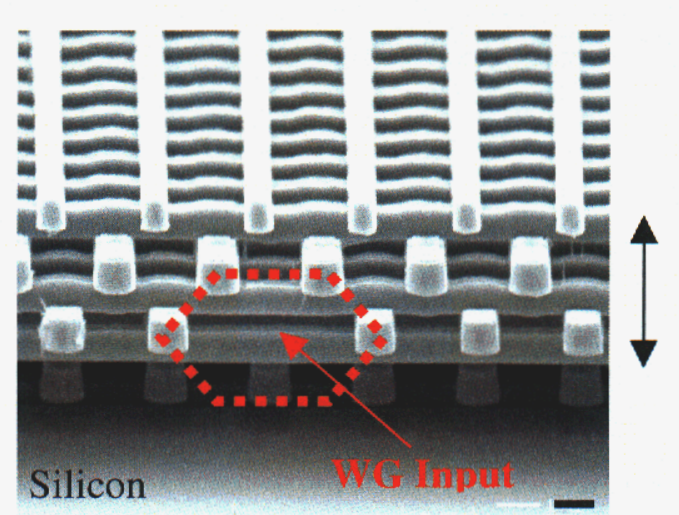

Figure 5. Oblique SEM image of a waveguide in a 3-D photonic lattice structure. The guide is formed by removing a rod from the structure.

$8 \mu \mathrm{m} \quad$ Waveguide bends were formed by removing rods on the row immediately above. The removed rods extended $1 / 4$ of a wavelength beyond the intersection. 


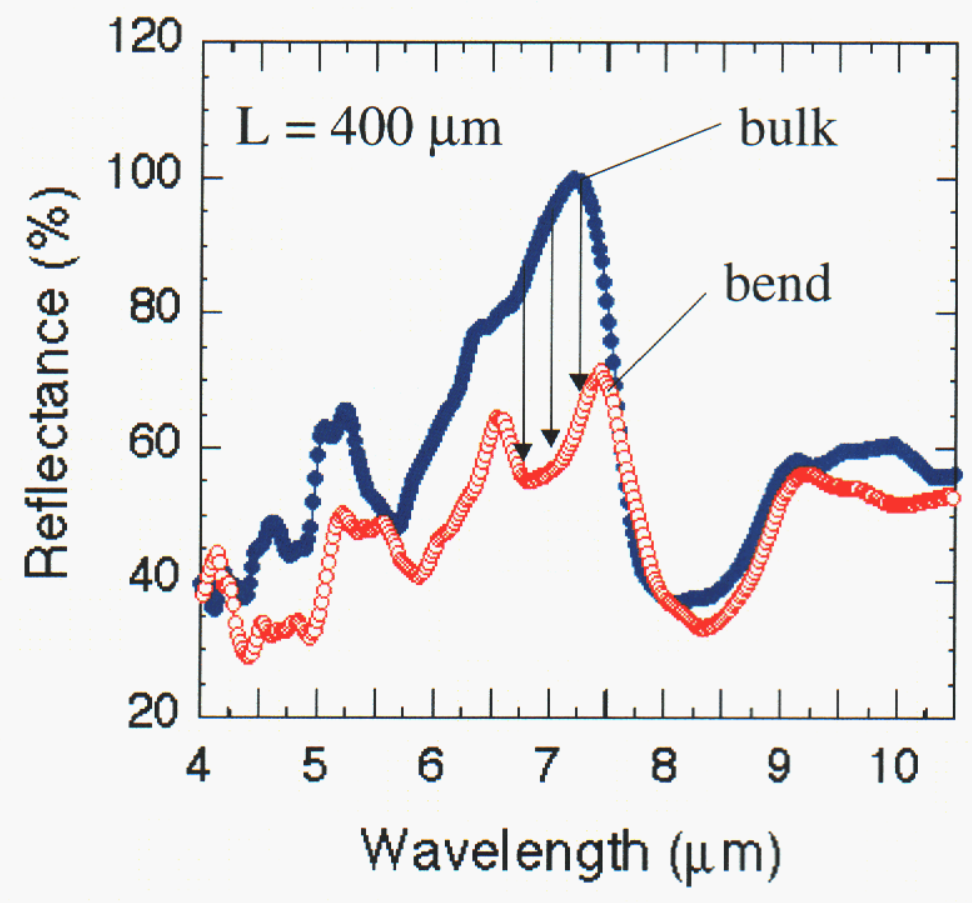

Figure 6. Evidence of waveguiding in the structure. The reflectance of $a$ region of the bulk crystal is compared with that in the vicinity of the waveguide. Wavelengths, which are not waveguided, are reflected. This indirect measurement is necessitated as a result of the relatively long wavelength of the bandgap. This was due to a tool limitation at the time of fabrication.

\section{DEMONSTRATION AND PROPERTIES OF BRAGG FIBERS}

As can be seen in the previous section, waveguiding in 3-D photonic lattice structures is possible, but the actual fabrication of such structures is complex. However, there is a simpler approach to this problem, which may have a similar level of functionality. The 3-D waveguide may be thought of as a hole surrounded by a omnidirectional mirror. A similar result can be obtained by imagining a high index contrast mirror structure, which is bent to form a tube through which light can propagate. This approach potentially enables the propagation of light in a low index material without the problem of the diffraction limit of light which forces mirror sizes to the 100's of micron in diameter and thereby increases the size and complexity of the resulting systems. If such a hollow mirror structure could be implemented and integrated with MEMS mirror arrays the mirrors and the system itself could be shrunk considerably. It turns out that the propagation of long wavelength, millimeter scale radiation through hollow waveguides is well known. Less well know are proposals in the 1960's to used hollow metal structures to propagate short wavelength light [1]. This field died out with the development of glass optical fibers. There is also interest in various coated hollow metal fibers for the propagation of 10 -micron radiation for medical applications [2-6]. The inside hollow diameter of these metallic structures is typically on the order of a millimeter. Yariv and coworkers first proposed Bragg fibers in 1978 [7]. Several other groups have recently revisited the idea of Bragg fibers [8-10]. In most cases the application being considered is that of optical fibers. Such fibers potentially have very low losses, in theory considerably less than that of standard silica fibers. However, this extremely low loss behavior is only exhibited by a particular mode, 
the $\mathrm{TE}_{01}$, which does not propagate in standard fibers. In our work we are interested in the development of miniature Bragg fibers with inner diameters in the order of 1-200 microns. These fibers are fabricated in the plane of the wafer using modifications of standard silicon processes. We are interested not in the very low loss applications associated with optical fiber communications, but rather applications associated with integrated optics and BioMEMS where losses on the order of $1-5 \mathrm{~dB} / \mathrm{cm}$ may be acceptable. Potential advantages of this approach in the field of integrated optics include: elimination of end facets, ease of integration with index matching and other fluids and possible development of novel thermo-optical structures. Potential advantages in the area of BioMEMS include the guiding of light and liquids in the same platform.

\section{BRAGG FIBER THEORY}

Typical waveguides guide light as the result of a difference in refractive index between two low loss optical media. Light is confined in such structures to the higher index media. The greater the index contrast between the low and high index media the greater the level of confinement. The greater the confinement the smaller the radius of curvature which can be defined without loss of light from the guide. The smaller the radius of curvature the smaller the size of the final part. However when light is tightly confined, its mode in the waveguide is very small and it becomes difficult to couple light into and out of the low index contrast optical fibers, which are the backbone of optical communications. The guiding principle of Bragg fibers is fundamentally different. In this case light propagates in a low index material, air, and is confined by a dielectric mirror. Compact bends can be realized using dielectric mirrors. The mode size for single mode propagation is on the order of a micron. However, in certain cases these structures can be made effectively single mode [9]. The efficiency of the mirror stacks used to confine the light is dependent on the index contrast between the high and low index materials making up the mirror. In the case of high index contrast systems such as silicon $(n \sim 3.5)$ and silicon dioxide $(\mathrm{n} \sim 1.46)$ only three pairs of layers result in reflectivity's greater than those of metals over a very large range of wavelengths. Also, in the case of these high index contrast systems, the mirrors are effective for all angles of incidence. The thickness of higher refractive index layers making up the mirror stack is very similar to those given by that of a 1/4-wave stack, the wavelength divided by four times the index of refraction. Optimal layer thickness targeted at a wavelength of 1.5 microns lower wavelength band edge, are 0.36 microns for $\mathrm{SiO}_{2}, 0.11$ microns for $\mathrm{Si}$ and 0.21 microns for $\mathrm{SiN}$. Such an arrangement results in low losses in the TE mode, but high losses for TM modes. TM mode losses can be reduced without greatly increasing TE mode losses by doubling the thickness of the inner most layer. Losses for these dimensions at 1.5 micron wavelengths for a 4 pair $\mathrm{Si} / \mathrm{SiO} 2$ mirror stack are predicted to be on the order of $3 \mathrm{~dB} / \mathrm{cm}$ for $\mathrm{TM}$ and $0.2 \mathrm{~dB} / \mathrm{cm}$ for TE. Losses can be reduced by increasing the number of layers and

can be greatly reduced by propagating only the $\mathrm{TE}_{01}$ mode in a $\sim 13$ micron diameter circular Bragg fiber [9].

\section{BRAGG FIBER EXPERIMENTAL DETAILS}

The structures were fabricated on 6-inch silicon wafers using widely available silicon processing tools. The process steps can generally be classified as the creation of an undercut trench, the sealing of the top of the trench, and the deposition of the mirror stack, Figure 7. The materials making up the mirror stack are introduced into the trench through periodic openings along it's length. The openings are widely enough spaced that they do not seem to effect the propagation of light along the trench to first 
order. The process relies on low sticking constant (high step coverage) of CVD (chemical vapor deposition) and diffusion processes, in particular the deposition of $\mathrm{Si}$ from silane, $\mathrm{SiN}$ from dichlorosilane and ammonia and the thermal oxidation of silicon. Combinations of processes enable the creation of $\mathrm{Si}, \mathrm{SiN}$ and $\mathrm{SiO}_{2}$ mirror layers. In Bragg fibers, the range of wavelengths propagated depends on the layer thickness. Since the thickness is determined by a CVD process we have very tight control over these parameters and it is readily possible to create waveguides which propagate short wavelength radiation. It is also noteworthy that no fine feature lithography is required to generate the structures. Relatively simple additions to the process can be employed to modify the tube shape, Figure 8 . Since the mirror levels are on the internal surfaces of the channel it is also possible to remove the opaque layers surrounding the structure, Figure 9.

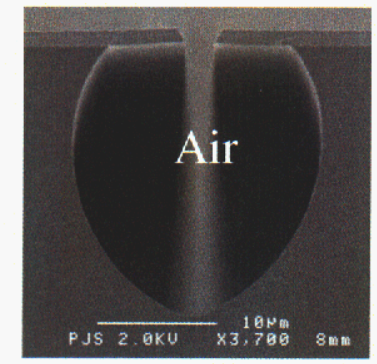

1a

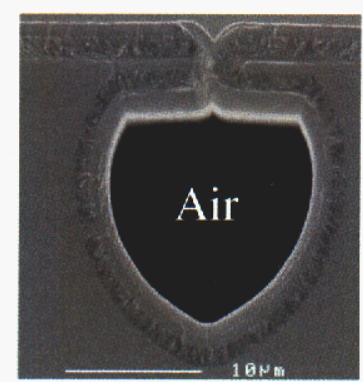

$1 \mathrm{c}$

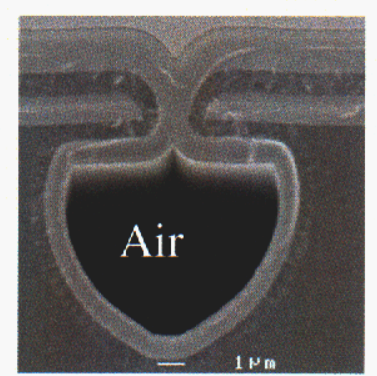

$1 \mathrm{~b}$

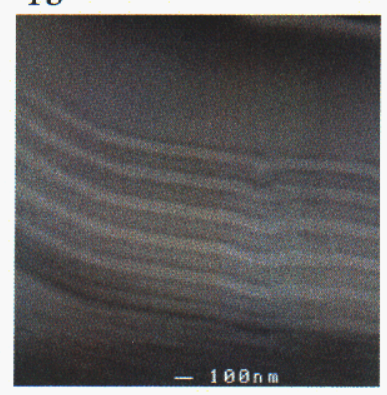

$1 \mathrm{~d}$
Figure 7. Scanning electron micrograph cross sections outlining the steps in the fabrication of miniature Bragg fibers. Ia shows the formation of the undercut trench. It is formed by isotropically etching the Si substrate through an open line in a low stress SiN layer. The isotropic etch is $F$ based. Figure $1 b$ shows the filling of the opening in the silicon nitride. This is achieved by first depositing 2 microns of polysilicon and then growing a 1-micron thermal oxide. Figure 1c shows the structure after the deposition of the mirror stack. Figure 1d shows a magnified image of a mirror stack consisting of $\mathrm{SiO}_{2}$ and $\mathrm{SiN}$ targeted at $400 \mathrm{~nm}$. The reactants needed for the deposition of the mirror stacks enter the trenches through periodic, larger openings not shown in this view, which are not closed off by the polysilicon and oxidation processes of step $b$. 

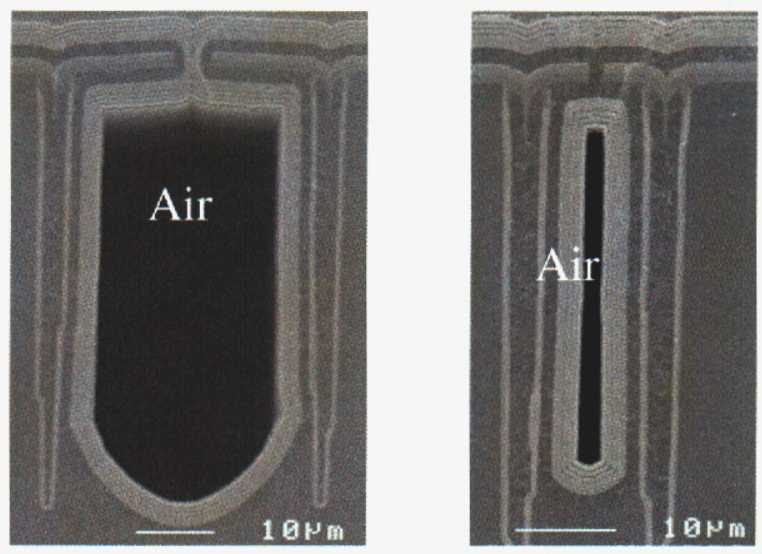

Figure 8. By adding a second mask level which is used to form oxide-lined trenches it is possible to control the lateral dimension of the trench. By controlling the width of the trench opening and the etch time it is possible to control the depth of the trench.

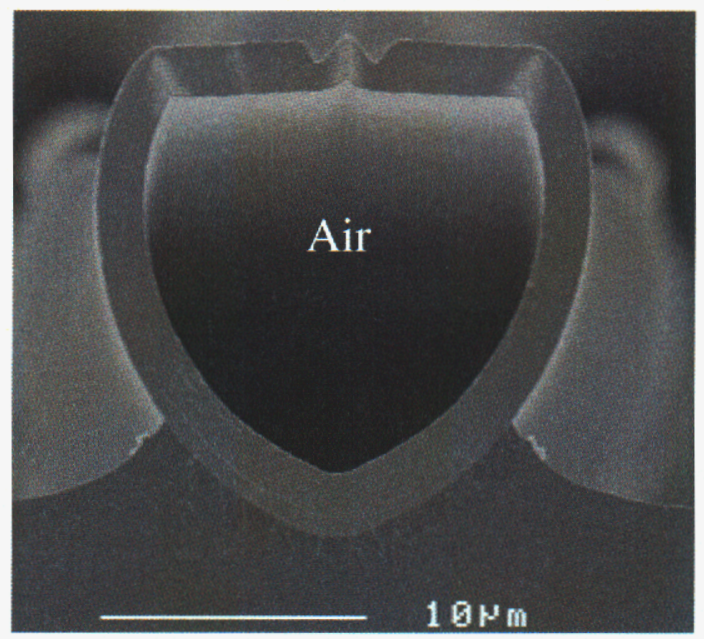

Figure 9. Since the mirror stack is located on the internal surfaces of the tube it is possible to blanket etch back the top layers to remove the optically opaque polysilicon layer used to close the top seam of the trench and, if desired, silicon from the trench edges. This will enable the propagation of light outside the "bandgap" of the mirror stack through the structure.

\section{BRAGG FIBER TESTING}

The optical properties of the structures were tested by the free space coupling of laser light into them. Tunable laser modules were used to investigate the wavelength dependence of light propagation through the channels. Losses were determined by investigating the variation of light intensity through various lengths of Bragg fiber. In the case of structures designed to propagate visible light, simple experiments were performed in which light from a tungsten white light source was propagated through the channels and the resulting image was captured on film. In all cases, the lengths of channel investigated ranged from 100's of microns to millimeters.

\section{BRAGG FIBER RESULTS AND DISCUSSION}


Figure 10 demonstrates the propagation of visible light through miniature Bragg fibers designed to have a short wavelength cutoff of $400 \mathrm{~nm}$. The light source was a white light tungsten bulb and the length of channel was roughly 6 millimeters.

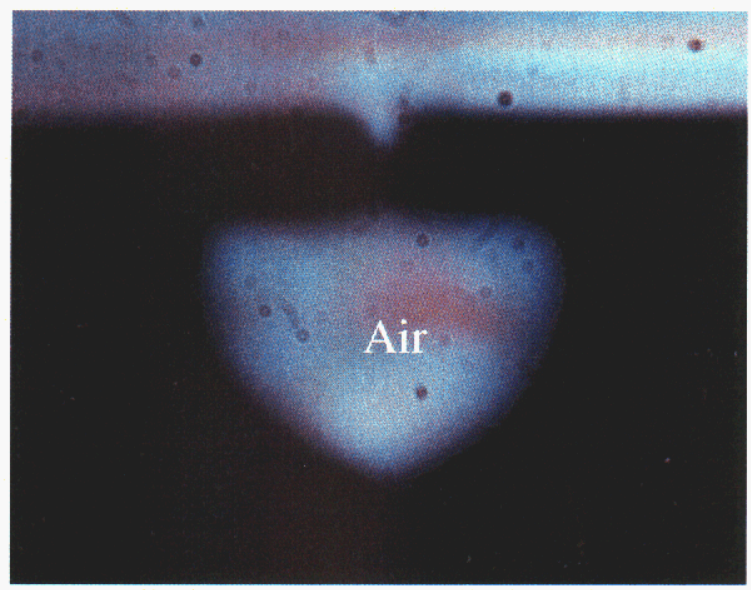

Figure 10. Optical image of visible light propagating through roughly 6 millimeters of a miniature Bragg fiber. The magnification is 1000 times. The Bragg fiber was targeted to have a lower wavelength cutoff at $400 \mathrm{~nm}$. The mirror stack consisted of $\mathrm{SiN}$ and $\mathrm{SiO}_{2}$.

Figure 11 shows a plot of light intensity as a function of wavelength for two different lengths of Bragg fiber with a lower wavelength edge at $\sim 1550 \mathrm{~nm}$. In this case the mirror layers were $\mathrm{Si}$ and $\mathrm{SiN}$ and the diameter was roughly 15 microns and there were 4 mirror pairs.

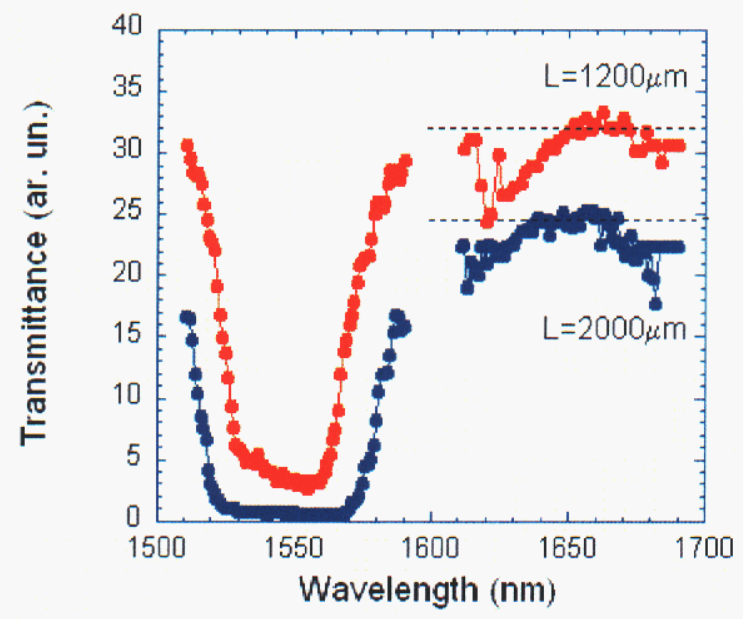

Figure 11. Transmission as a function of wavelength for two different lengths of Bragg fiber. The lower band edge is at 1550 nm. The discontinuity at $\sim 1600 \mathrm{~nm}$ is the result of changing laser modules. The exact long wavelength cutoff is unknown since the series of laser modules available did not extend further than $1680 \mathrm{~nm}$.

From data similar to that presented in Figure 11 we estimate the losses in these structures to be on the order of $2-5 \mathrm{db} / \mathrm{cm}$. This is in good agreement with that predicted by theory. The losses of the shorter wavelength structures of Figure 10 have not yet been measured, however, we believe the losses will be considerably lower. Modeling indicates that much of the loss in $\mathrm{Si} / \mathrm{SiN}$ mirror stacks is the result of adsorption by the polysilicon in the mirror stack. The value of polysilicon adsorption used in the theory was $10 \mathrm{~dB} / \mathrm{cm}$. The adsorption coefficients of silicon dioxide and silicon nitride are much lower.

Since the range of transmission of the structures exceeded the range of the available turnable laser modules we were unable to accurately identify the full bandwidth of the Bragg fibers tested in Figure 11. 
However, the $100 \mathrm{~nm}$ demonstrated is more than adequate for many applications. It is also notable that the drop off of intensity at $\sim 1550 \mathrm{~nm}$ evident in Figure 11 is very sharp.

Since the light in these structures is propagating in air there is no discontinuity in index as the light from the laser enters and leaves the Bragg fiber. As a result the Fabry-Perot resonant features typically present are, in this case, entirely absent.

We have observed experimentally that the coupling of laser light into the miniature Bragg fibers is relatively insensitive to the alignment of the beam with the centerline of the fiber, making laser coupling to the Bragg fibers relatively straightforward. Theory predicts that, for the Bragg fibers to be single mode, the diameter of the tube should be on the order of a micron. While this should be possible it may be relatively difficult to achieve with the current fabrication process. The roughly 15 micron diameter structures fabricated to date are multimode. It has been theoretically predicted that circular structures with similar dimensions could be made to be effectively single mode. Losses of the $\mathrm{TE}_{01}$ mode are predicted to be significantly less than that of other modes [9]. Thus during propagation over long distances all the other modes will dissipate leaving only the $\mathrm{TE}_{01}$ mode. It should be possible to fabricate quite circular miniature Bragg fiber structures; however, this mode is not typically generated by light emitting devices. While the integrated mode size of the miniature Bragg fibers can be made to match that of a single mode fiber the actual mode shape will be different. This may be an issue for integrated optics applications. We are in the process of experimentally determining what effect this has on the input and output coupling of such structures to fibers and whether or not it is possible to geometrically come up with structures which will only support a single mode.

We have also investigated the formation of both splitters and bends, which will be important building blocks of any real device. Both have been demonstrated. Bends with radii of 40 microns have less than $0.4 \mathrm{~dB}$ loss per 90 -degree bend. Mirror bends taking up roughly the same area have similar performance.

The presence of the gas inlet ports along the structure at intervals ranging from 400 to 1600 microns did not appear to have a major influence on loss. This is not unexpected since the openings are well spaced and the "air gaps" are roughly the same size as the hollow tubes.

\section{POTENTIAL APPLICATIONS OF BRAGG FIBERS}

While these structures are still being evaluated, we believe that they may have many potential applications to integrated optics and BioMEMS. One of the major issues in large optical switching arrays is the divergence of light as it propagates in air. This leads to large mirror sizes and requires collection, focusing, alignment and collimator schemes. In miniature Bragg fibers, light also propagates in air, however the light is confined by the Bragg fiber. It may be possible to integrate an array of crossing Bragg fibers with a series of movable, two-position mirror MEMS devices. The MEMS mirrors could fit into the gas inlet ports. In this $\mathrm{N}^{2}$ structure the light would be confined to the Bragg fiber. The mirrors would therefore only need to be on the order of the diameter of the fiber, 5-10 microns. It may even be possible to fabricate very large, $\sim 125$ micron diameter, Bragg fibers into which the optical fiber can be inserted. The fibers can then be adiabatically tapered down to 5 micron in diameter. This approach 
would eliminate the need for collimators, lensing and other fiber alignment structures. Components of such a system are currently in development.

At present, we have only demonstrated the propagation of light in "air-filled" Bragg fibers. However, it should also be possible to introduce low index liquids into such structures. Many potentially interesting fluids exist. For example, certain fluids exhibit changes of index with temperature, which are an order of magnitude greater than those of solids [11]. It may be possible to integrate fluid filled Bragg fibers with heaters to make more efficient thermo-optical devices such as switches and attenuators.

Finally, we believe that it may be possible to fill such structures with biological fluids. In these structures both light and liquids would be guided within a single platform. At present there exist conventional structures where light, confined in dielectric waveguides, is evanescently coupled into fluorescently tagged biological samples [12-13]. Light emitted from the tags is then evanescently coupled back into the waveguide. Both of these evanescent coupling processes are relatively inefficient. On the other hand if light and fluid could be confined to a single channel the interaction of light with the liquid and the trapping of the emitted light in the channel could be much more efficient.

\section{SUMMARY AND CONCLUSIONS}

We have demonstrated the feasibility of fabricating high quality 1-D photonic lattice mirror structures using Si processing tools over wavelengths ranging from $400 \mathrm{~nm}$ to $1700 \mathrm{~nm}$. We have integrated these with SUMMiT ${ }^{\mathrm{TM}}$ structures. The major problem encountered being stress induced mirror curvature, which we believe could be addressed by changes in design or changes in the anneal process. We have also demonstrated switchable cavity structures of a variety of configurations.

Miniature Bragg fibers have been demonstrated for the first time. The structures are fabricated using standard silicon processing tools on 6-inch wafers. A wide range of different geometry of structure has been demonstrated as have a number of different mirror stack configurations. Guiding of light from $\sim 400$ to $1700 \mathrm{~nm}$ has been demonstrated as have bends and splitters. Losses are $2-5 \mathrm{~dB} / \mathrm{cm}$ and potentially can be many orders of magnitude lower. There are many potentially interesting applications for these novel structures.

\section{REFERENCES}

1. E.A.J. Marcatili and R.A. Schmeltzer, "Hollow Metallic and Dielectric Waveguides for Long Distance Optical Transmission and Lasers", Bell System Tech. J., July (1964), 1783.

2. M. Miyagi and S. Kawakami, "Design Theory of Dielectric-Coated Circular Metallic Waveguides for Infrared Transmission", J. Lightwave Tech., LT-2, 116 (1984).

3. N. Croitoru, J. Dror and I. Gannot, "Characterization of hollow fibers for the transmission of infrared radiation", Appl. Optics, 29, 1805 (1990).

4. Y. Kato and M. Miyagi, "Modes and Attenuation Constants in Circular Hollow Waveguides with Small Core Diameters for the Infrared", IEEE Trans. Microwave Theory and Tech., 40, 679 (1992). 
5. F.E. Vermeulen, T. Wang C.R.James and A.M. Robinson, "Propagation of Infrared Radiation in Hollow Microstructural Cylindrical Waveguides", J. Lightwave Tech., 11, 1956 (1993).

6. R. L. Kozodoy, A.T. Pagkalinawan and J. A. Harrington, "Small-bore hollow waveguides for delivery of 3- $\square$ m laser radiation", Appl. Optics, 35, 1077 (1996).

7. P.Yeh, A. Yariv and E. Marom, "Theory of Bragg fiber", J. Opt. Soc. Am. 68, 1196 (1978).

8. Y. Xu, R.K. Lee and A. Yariv, "Asymptotic analysis of Bragg fibers", Optics Lett., 25, 1756 (2000).

9. S.G. Johnson et-al, "Low-loss asymptotically single-mode propagation in large-core OmniGuide fibers", Optics Exp., 9, 748 (2001).

10. T. Kawanishi and M. Izutsu, "Coaxial periodic optical waveguide", Optics Exp., 7, 10 (2000).

11. M.B.J. Diemeer, J.J. Brons and E.S. Trommel, "Polymeric optical waveguide switch using the thermooptic effect", J. Lightwave Tech., 7, 449 (1989).

12. T.R.E. Simpson, D.J. Revell, M.J. Cook and D.A. Russell, Evanescent Wave Exited Fluorescence from Self-Assembled Phthalocyanine Monolayers", Langmuir, 13, 460 (1997).

13. H.P. Kao and J.S. Schoeniger, "Hollow cylindrical waveguides for use as evanescent fluorescencebased sensors: effect of numerical aperture on collected signal", Appl. Optics, 36, 8199 (1997). 
DISTRIBUTION:

$1 \quad$ MS $1080 \quad$ S.S. Mani, 1749

1 MS $1080 \quad$ H.D. Stewart, 1749

1 MS 9018 Central Technical Files, 8945-1

2 MS 0899 Technical Library, 9616

1 MS 0612 Review \& Approval Desk, 9612

1 MS $0188 \quad$ D. Chavez, LDRD Office, 1030 